Z. EEG-EMG 18 (1987) 131-134

(C) Georg Thieme Verlag Stuttgart - New York

\title{
Grenzzoneninfarkte mit symptomatischer Epilepsie - klinische und elektroenzephalographische Befunde
}

\author{
P. Berlit, B. Bühler, K.-H. Krause*
}

Neurologische Kliniken Mannheim und Heidelberg * der Universität Heidelberg

\section{Borderzone infarctions with seizures - Clinical and elec- troencephalographic findings}

\begin{abstract}
In 6 patients with repeating seizures early after stroke periodic lateralizing epileptiform discharges were detected in EEG. Computed tomography scans showed borderzone infarctions including the territories of the middle and the posterior cerebral artery in four patients; sonographic and angiographic findings were suspicious of embolic stroke in five cases. Seizures and EEG-discharges resolved during a short term treatment with anticonvulsants. In contrast to cerebral infarctions of other regions with comparable EEG-changings long term treatment was necessary in none of the patients.
\end{abstract}

Key-Words: Borderzone infarction -- Periodic epilepti form discharges - Vascular epilepsy

\section{Zusammenfassung}

Bei sechs Patienten mit seriell auftretenden epileptischen Anfällen im Rahmen eines frischen Hirninfarktes werden periodisch auftretende einseitige Spitzenentladungen im EEG beschrieben. Bei vier Kranken ließen sich computertomographisch Infarktbezirke im Grenzzonenbereich zwischen dem Versorgungsgebiet von mittlerer und hinterer Hirnarterie nachweisen. Dopplersonographische und angiographische Befunde sprachen bei fünf Patienten für eine embolische Genese der Infarzierung. Unter einer vorübergehenden antikonvulsiven Therapie bildeten sich die epileptischen Anfälle und die EEG-Veränderungen innerhalb von Stunden zurück. Eine Dauertherapie war im Gegensatz zu entsprechenden EEG-Veränderungen bei Großhirninfarkten anderer Lokalisation in keinem Fall erforderlich.

ämie. Bei diesen Patienten beginnt das Krankheitsbild mit einer Serie oder einem Status fokaler Anfälle, bei deren $\mathrm{Ab}$. klingen eine Hemiparese der betroffenen Extremitäten resultiert. Da es sich bei den entsprechenden Kasuistiken jeweils um akute hypoxische Ereignisse handelt, wird ursächlich eine vorübergehende ischämiebedingte erhöhte zerebrale Anfallsbereitschaft im Randbezirk angenommen (11). In der Regel ist das Grenzgebiet zwischen dem Versorgungsbereich der A. cerebri posterior und dem der A. cerebri media betroffen. Die stets passageren krisenhaften EEG-Veränderungen müssen sich klinisch nicht immer in epileptischen Syndromen manifestieren, entsprechende Befunde werden auch subklinisch und bei nicht epileptischen Manifestationen (z. B. der Basilaris-Migräne) beschrieben (1). Die Autoren verwandten für ihre klinischen und elektroenzephalographischen Beobachtungen den Begriff des „Grenzlandes der Epilepsie".

Im folgenden wollen wir über sechs Patienten berichten, die wir in den letzten zwei Jahren zu (iesicht bekamen und die die typischen, periodischen, okzipital lokalisierten EEGEntladungen im Rahmen eines ischämischen Ereignisses zeigten.

\section{Patienten und Methodik}

Bei Patienten, die mit einem akuten ischämischen Ereignis stationär aufgenommen wurden und während der Akutphase fokale oder generalisierte epileptische Anfälle boten, wurde zum frühestmöglichen Zeitpunkt, spätestens jedoch 
innerhalb von 24 Stunden, ein Elektroenzephalogramm abgeleitet. Die Ableitung erfolgte mit cinem Schwarzer-12Kanal-FEC-Cierät in Standardschaltungen gegen das gleichund gegenseitige Ohr als Referencelektrode sowie in Längs- und Querreihenmontagen. Innerhalb der ersten Woche nach dem akuten Ereignis erfolgte eine computertomographische Untersuchung des Schädeis nit axialer Schichtfuihrung sowie eine dopplersonographische Untersuchung der hirnversorgenden extrakraniellen Gefäßße. In Abhängigkeit von möglichen therapeutischen Konsequenzen wurde cine angiographische Darstellung der Hirngeräkse veranlakt.

Diejenigen Patienten, die im initial angefertigten Elektroenzephalogramm die von Gastaut u. Naquet (9) beschrieben periodischen okzipital lokalisierten Spitzenentladungen boten, wurden in die weitere Auswertung miteinbez.ogen.

\section{Ergebnisse}

Die wesentlichen klinischen Daten unserer sechs Patienten sind in Tab. 1 zusammengestellt. Es handelt sich um vier männliche und zwei weibliche Kranke im Alter zwischen 51 und 84 Jahren, vier Patienten waren über 75 Jahre alt. In jedem Falle bestand klinisch das Bild epileptischer Anfälle, wobei fünfmal die Epilepsie Initialsymptom des ischämischen Ereignisses war; bei einer Patientin führte zwar ein Hemi-Grand mal zur Aufnahme in die Klinik, das CT zeigte jedoch einen bereits älteren Posteriorinfarkt und auch im Verlauf keine frischen Ischämiezonen. Mit Aus- nahme dieser Patientin handelte es sich stets um in Seric oder in Form eines Status auftretende epileptische Anfälle, wobei einmal das Bild einer Epilepsia partialis continua Kozevnikov imponicrte, dreimal motorische Jackson-Anfalle und einmal Grand mal in Serie vorlagen. Bei jedem der Kranken bestand eine Gesichtsfeldeinschränkung entweder in Form einer homonymen Hemianopsie zur Gegenscitc oder einer hemianopischen Aufmerksamkeitsschwäche. Bei einer Patientin, die bei Aufnahme komatös war, wurde die Hemianopsie später bestätigt. Das Computertomogramm zeigte einen frischen Infarkt bei vier Patienten. Darüber hinaus bestanden älterc Ischämiebezirke bei drei Kranken. Die akuten Ischämien betrafen entweder das Versorgungsgebiet $\operatorname{der} \mathbf{A}$. cerebri posterior, wobei immer die Grenze zum Media-Versorgungsgebiet erreicht oder auch leicht überschritten wurde (Abb. 1). Zweimal lag ein Mediateilinfarkt vor, der das Grenzgebiet zur hinteren Hirnarteric erreichte. Einen hämodynamisch relevanten, stenosierenden Prozeß der extrakraniellen, hirnversorgenden Gefäße sahen wir nur bei einem Patienten; bei dem mit 51 Jahren jüngsten Patienten zeigtc dic Angiographie eine Zirkulationsstörung im Bereich der korrespondierenden mittleren Hirnarterie, ein Befund, der typisch für embolisch bedingte Infarkte ist (3). Bei den restlichen vier Kranken ergab die dopplersonographische Untersuchung der Halsgefäße einen unauffälligen Befund. Aufgrund des fortgeschrittenen Alters wurde bei diesen Patienten keine Angiographie durchgeführt. Wir werten die negative Dopplersonographie an ehesten als Hinweis auf eine emboli. sche Cicnese des Infarktcs, zumal bei zwei dieser Kranken

Tab. 1 Befunde bei sechs Patienten mit periodischen paroxysmalen EEG-Veränderungen bei Grenzzonen-Infarkten im Aa. media/posterior-Bereich

\begin{tabular}{|c|c|c|c|c|}
\hline $\begin{array}{l}\text { Alter } \\
\text { Geschlecht }\end{array}$ & Klinik & Computertomographie & Gefäßstatus & Risikofaktoren \\
\hline $69 m$ & $\begin{array}{l}\text { Epilepsia partialis continua } \\
\text { links in Arm und Gesicht. Ho- } \\
\text { monyme Hemianopsie nach links. } \\
\text { Monoparese im linken Arm }\end{array}$ & Status lacunaris & $\begin{array}{l}\text { Interna-Abgangs-Stenose } \\
80 \% \text { rechts (Doppler- } \\
\text { sonographie) }\end{array}$ & $\begin{array}{l}\text { Hypertonus } \\
\text { absolute Arrhythmie }\end{array}$ \\
\hline $51 \mathrm{~m}$ & $\begin{array}{l}\text { motorische Jackson. Anfälle im } \\
\text { rechten Arm. Gemischte Aphasie. } \\
\text { Hemianopische Aufmerksamkeits- } \\
\text { schwäche nach rechts. Sensomo- } \\
\text { torische Hemiparese rechts }\end{array}$ & $\begin{array}{l}\text { Mediateilinfarkt } \\
\text { links }\end{array}$ & $\begin{array}{l}\text { Zirkulationsstörung in } \\
\text { der A. cerebri media } \\
\text { links (Angiographie) }\end{array}$ & Hypertonus \\
\hline $82 m$ & $\begin{array}{l}\text { Grand mal-Serie. Kortikale } \\
\text { Blindheit. Alte Hemiparese } \\
\text { links }\end{array}$ & $\begin{array}{l}\text { frischer Posterior- } \\
\text { infarkt links. Alter } \\
\text { Posteriorinfarkt } \\
\text { rechts }\end{array}$ & $\begin{array}{l}\text { normal (Dopplersonogra- } \\
\text { phie) }\end{array}$ & Hypertonus \\
\hline $84 w$ & $\begin{array}{l}\text { motorische Jackson-Anfälle im } \\
\text { linken Arm und Gesicht. Devia- } \\
\text { tion conjugée nach links. He- } \\
\text { miparese links, Koma }\end{array}$ & $\begin{array}{l}\text { Mediateilinfarkt } \\
\text { rechts }\end{array}$ & $\begin{array}{l}\text { normal (Dopplersonogra- } \\
\text { phie) }\end{array}$ & $\begin{array}{l}\text { Hypertonus } \\
\text { absolute Arrhythmie }\end{array}$ \\
\hline $83 m$ & $\begin{array}{l}\text { motorische Jackson-Anfälle im } \\
\text { linken Arm und Gesicht. Hemi- } \\
\text { anopische Aufmerksamkeits- } \\
\text { schwäche nach links. Monopare- } \\
\text { se im linken Arm }\end{array}$ & $\begin{array}{l}\text { frischer Grenzzonen- } \\
\text { infarkt im Media/ } \\
\text { Posterior-Bereich } \\
\text { rechts. Alterer Po- } \\
\text { steriorinfarkt links }\end{array}$ & $\begin{array}{l}\text { normal (Dopplersonogra- } \\
\text { phie) }\end{array}$ & $\begin{array}{l}\text { Hypertonus } \\
\text { Diabetes mellitus }\end{array}$ \\
\hline $76 w$ & $\begin{array}{l}\text { Halbseitenanfall links. Homo- } \\
\text { nyme Hemianopsie nach links. } \\
\text { Muskeleigenreflexe links aus- } \\
\text { geprägter als rechts }\end{array}$ & $\begin{array}{l}\text { älterer Posterior- } \\
\text { infarkt rechts }\end{array}$ & $\begin{array}{l}\text { normal (Dopplersonogra- } \\
\text { phie) }\end{array}$ & $\begin{array}{l}\text { Hypertonus } \\
\text { absolute Arrhythmie }\end{array}$ \\
\hline
\end{tabular}




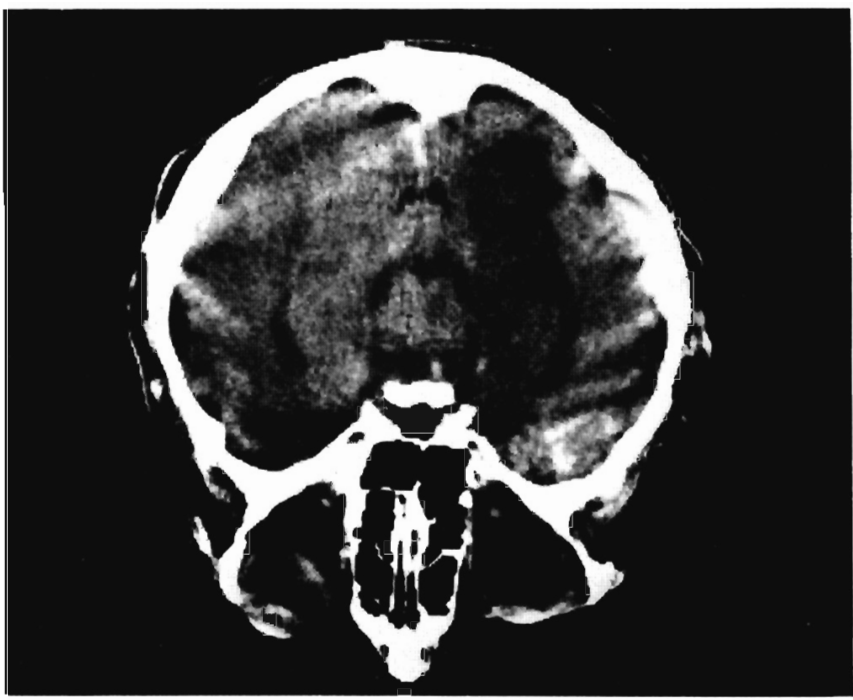

Abb. 1 Computertomogramm eines 83jährigen Patienten mit Grenzzoneninfarkt im Media/Posterior-Bereich (zwei Tage nach Auftreten von motorischen Jackson-Anfällen des linken Armes und Gesichts und einer homonymen Hemianopsie nach links). Beach te die Miteinbeziehung temporo-okzipitaler und basaler Strukturen.

eine absolute Arrhythmie des Herzens vorlag. Darüber hinaus fanden sich an Gefäßrisikofaktoren bei jedem der sechs Kranken ein erhöhter Blutdruck und einmal ein Diabetes mellitus.

Im Elektroenzephalogramm, das jeweils am Tag des akuten Ereignisses angefertigt wurde, zeigten sich die charakteristischen periodischen, fokalen Entladungen über der Okzipitalregion mit Ausbreitung nach parietal und temporal; es handelte sich dabei um Spitzenpotentiale und Sharp waves, die sich auf die auch computertomographisch betroffene Hemisphäre beschränkten (Abb. 2). Die epileptischen Entladungen korrespondierten bei den vier Patienten mit fokaler Epilepsie mit den klinischen Symptomen; bei den zwei Kranken mit generalisierten Anfällen waren sie auch im Intervall nachweisbar. Die Behandlung erfolgte bei fünf Kranken mit Diphenylhydantoin, ein Patient erhielt zusätzlich, ein anderer ausschließlich Clonazepam parenteral in individueller Dosierung. Unter der angegebenen Medikation wurde stets innerhalb von maximal zwei Stunden Anfallsfreiheit erzielt. Die jeweils 24-72 Stunden nach dem ersten EEG abgeleitete Kontrolle zeigte zwar stets eine fokale Verlangsamung, jedoch nie die zuvor nachgewiesenen epilepsietypischen Entladungen.

Während sich die sensomotorische Halbseitensymptomatik der Gegenseite in der Regel mehr oder weniger befriedigend besserte, blieb mit einer Ausnahme die akut aufgetretene Gesichtsfeldstörung bei unseren Kranken bestehen. Bei einem Patienten resultierte bei einem computertomographisch nachweisbaren alten Posteriorinfarkt durch die frische Infarzierung eine kortikale Blindheit. Bei drei unserer Kranken, deren Verlauf wir weiter verfolgen konnten, traten auch ohne entsprechende Behandlung keine weiteren Anfälle auf.

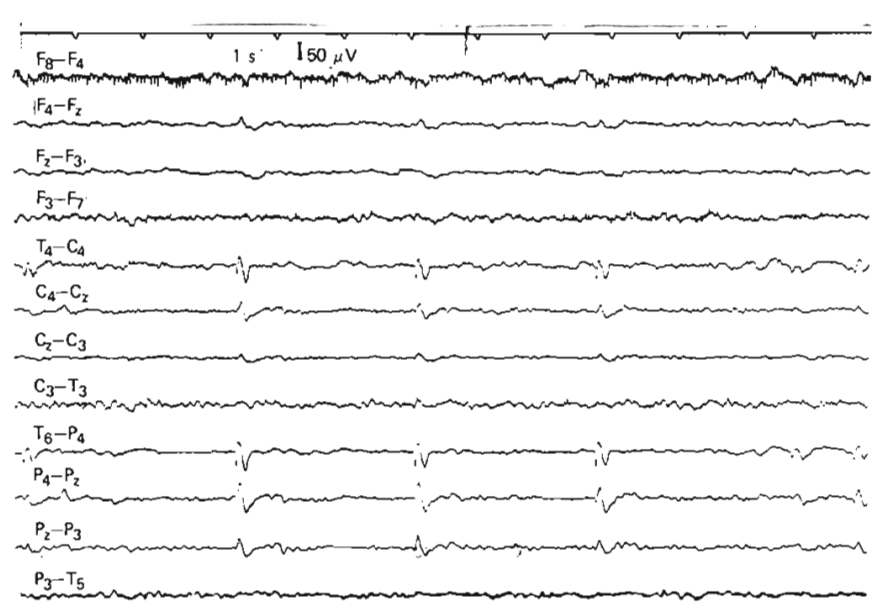

Abb. 2 EEG.Befund des Patienten aus Abb. 1, vier Stunden nach der stationären Aufnahme. Zum Zeitpunkt der Ableitung bestehen fokale Zuckungen des linken Armes und der linken Gesichtshälfte.

\section{Diskussion}

Bei Hirninfarkten im Grenzbereich der Versorgungsgebiete zweier Arterien kann es sich um sogenannte Wasserscheideninfarkte handeln, die auf dem Boden hämodynamischer Veränderungen im Endstrombereich des jeweils betroffenen Gefäßes auftreten. Es kann aber auch ein Territorialinfarkt vorliegen, der -- meist embolischer Genese -- das Teilversorgungsgebiet eines der zerebralen Gefäße erfaßt. Treten im Rahmen eines Hirninfarktes epileptische Anfälle auf, so handelt es sich computertomographisch zumeist um Territorialinfarkte; bei Wasserscheideninfarkten scheinen fokale oder generalisierte Anfälle nur sehr selten vorzukommen. Dies scheint auch für den Grenzbereich zwischen den Versorgungsgebieten der A. cerebri media und posterior zu gelten; während Bogousslavsky und Regli (4) bei elf Wasserscheideninfarkten dieser Lokalisation keine epileptischen Anfälle beobachten konnte, handelte es sich bei vier unserer Kranken durchweg um grenzüberschreitende Territorialinfarzierungen.

Interessant ist aus klinischer Sicht die Neigung zum Auftreten von - zumeist fokalen -- Anfällen in Serie. Elektroenzephalographisch ist das Kurvenbild bestimmt durch die periodisch auftretenden epileptischen Entladungen, die auf die betroffene Hemisphäre beschränkt sind. In Übereinstimmung mit einer großen Zahl anderer Autoren $(1,2,5,6)$ treten die im angloamerikanischen als periodische lateralisierte epileptiforme Entladungen bezeichneten Transienten bevorzugt bei hinteren Grenzzoneninfarkten auf; entsprechende - häufig allerdings nicht periodisch auftretende Spitzenpotentiale können jedoch auch bei Infarkten anderer Lokalisationen (6) oder sogar bilateral bei zusätzlicher generalisierter Hirnschädigung durch eine Hypoxie (7) vorliegen. Ursache für. die bevorzugte Manifestation im Poste- 
rior-Media-Grenzgebiet könnte die Einbeziehung von Anteilen des Gyrus temporo-occipitalis und Gyrus hippocampus sein, welche bei zwei unserer Patienten auch computertomographisch erkennbar war (Abb. 2). Neben der Lokalisa. tion des Infarktes könnte auch die Akuität des Eintretens der Ischämie bei embolischer Genese, die bei funf unserer sechs Kranken anzunehmen ist, eine Rolle spielen. Während Holmes (10) bei sechs Hirninfarktpatienten mit periodischen Spitzenentladungen, bei denen nicht ausschließlich das Grenzgebiet Media-Posterior betroffen war, in jedem Fall die Entwicklung einer vaskulären Epilepsie beobachtete, war bei unseren Kranken dic Prognose im Hinblick auf die Anfälie durchweg günstig, so daß wir eine antikonvulsive Langzeitmedikation bei diesen Kranken nicht für gerechtfertigt erachten.

Eine interessante Beobachtung teilten Verma und Kooi (16) nit. Diese Autoren fanden bei 15 Kranken mit einem Hirninfarkt kontralateral aperiodische, bis zu drei Monater nach dem Ereignis persistierende, epilepiforme Transienten, welche durchweg nicht mit klinischen Anfällen assoziiert waren. Bei diesen elektroenzephalographischen Phänome. nen scheint ebenfalls eine generelle Hirnschädigung eine

\section{Literatur}

1 Barolin, G.S., K. Karbowski: Okzipitale Krisen im ,Grenzland der Epilepsie". Z. EEG-EMG 4 (1973) 1-8

2 Barolin, G.S., E. Scherzer, R. Naquet, H. Gastaut: Etude électroclinique des ",tats de mal" épileptiques survenant chez les apoplectiques. Rev. neurol. 107 (1962) 242-249

3 Berlit, P.: Die kardiale Hirnembolie. Nervenarzt 54 (1983) 389 399

4 Beygousslavsky, J., F. Regli: Burderzone Infarctions distal to internal carotid artery occlusion: prognostic implications. Ann. neurol. 20 (1986) 346-350

5 Chatrian, G.E., C.M. Shaw, H. Leffman: The significance of periodic lateralized epileptiform discharges in EEG. Electroenceph. Clin. Neurophysiol. 17 (1964) $177-193$

6 Christian, $W .:$ Klinische Flektroencephalographie 3. Aufl. Thiemc, Stuttgart, Ncw York 1983

7 de la $P a z$ D., R.P. Brenner: Bilateral independent periodic lateralized epileptiform discharges. Arch. Neurol. 38 (1981) 713 715

8 Enge, S., K. Kömer, S. Fuchs, G. Ladurner, G. Schneider, II. Lechner: Elcktroenzephalographische und computertomographische Befunde bei vaskulïrer Epilepsie ischämischer Cenese. Z. EEG-EMG 14 (1983) 191194

9 Gastaut, H., R. Naquet: Etude électroencéphajographique d l'insuffisance circulatoire cérébrale. In: Symposium Inter- . national sur la Circulation cérébrale, 163-191. Editions Sandoz, Paris 1966

10 Holmes, G.L.: The Electrocnecphalogram as a Predictor of Sei-
Rolle zu spielen, ähnlich wie bei den bilaterai auftretenden periodischen Entladungen.

Faßt man die elektroenzephalographischen Beobachtungen zusammen, ergibt sich folgendes Bild: wenn es beim akuten Hirninfarkt zum Auftreten von periodischen epileptischen Entladungen auf der Seite eines computertomographisch nachweisbaren Grenzzoneninfarktes Media/Posterior kommt, ist lediglich eine vorübergehende antikonvulsive Behandlung der zumeist in Serie auftretenden fokalen Anfalle indiziert -- eine Langzeittherapie ist nicht erforderlich. Treten periodisch oder nichtperiodisch epilepsietypische Potentiale auf der Seite eines Großhirninfarktes anderer Lokalisation oder bilateral auf, muß mit der Entwicklung einer vaskulären Epilepsie gerechnet werden; eine antikonvulsive Dauertherapie ist angezeigt.

Finden sich nichtperiodische epilepsietypische Entladungen ausschließlich kontralateral zum Hirninfarkt, ist eine Gabe von Antiepileptika nicht indiziert: es sollte jedoch eine eingehende internistische Diagnostik und Thcrapie bei Verdacht auf generalisierte (z. B. hypoxische) Hirnschädigung erfolgen. zures Following Cerebral infarction. Clin. Ejectroenceph. 11 (1980) 83-86

11 Karbow'ski, K.: Fokale periodische Spitzenpotentiale bei extraterriorieller zerebraler Ischämic. Z. LEG -LMG 6 (1975) 27--31

12 Kehrer, H.E., K. Aretz: Epileptische Anfälle bei zerebraler Gefäßsklerose. Psychiatr. Neurol. (Basel) 16 (1963) 265-271

13 Logar, C., R. Martischnig, S. Enge, W.D. Sager, G. Ladurner: Zur Wertigkeit von EEG und Computertomographie bei ischümischen Insulten. Z. EEG-EMG 10 (1979) 161-166

14 Liihdorf. K., I.K. Jensen, A.M. Plesner: The value of EEG in the investigation of postapoplectic cpilcpsy. Acta Neurol. Scand. 74 (1986) $279-283$

15 McDowell. F. C,E. Wells. C. Ehlers: The electroencephalogram in internal carotid artery occlusion. Neurology (Minn.) 9 (1959) $678-681$

16 Verma N.P., K.A. Kooi: Contralateral Epileptiform Transients in Stroke (CETS). Epilepsia 27 (1986) 437-440

P. Berlit, B. Bühler, K.-H. Krause: Grenzzoneninfarkte mit symptomatischer Epilepsie klinische und clcktroenzephalographische Befunde. Z. EEG-I:MG 18 (1987) 131-134

Anschrift

Priv. Doz. Dr. P. Berlit, Neurol. Universitätsklinik, Theodor-KutzerUfer, D-6800 Mannheim 1 